\title{
Memorias de militantes feministas en la dictadura y en la apertura política en Brasil: la cuestión del trabajo doméstico como reivindicación democrática (1964-1990)
}

\author{
Soraia Carolina de Mello \\ Universidad Federal de Santa Catarina (UFSC), Instituto de Estudios de Género (IEG), Brasil \\ soraiaa.mello@gmail.com
}

\section{Resumen:}

Este artículo utiliza como fuente memorias de mujeres que fueron militantes feministas durante la dictadura militar en Brasil y que también participaron de la apertura política en el país, a partir de mediados de los años 1980. A través de ellas, se investiga, desde el punto de vista de esas mujeres, comprometidas políticamente con los feminismos y muchas veces con las izquierdas, las interlocuciones entre las problematizaciones feministas acerca del trabajo doméstico y la perspectiva de sociedad democrática que se abre durante el período. Bajo el slogan "democracia en el país y en la casa", los debates feministas articularon lo público y lo privado al denunciar la carga de trabajo doméstico, que era ejecutada casi exclusivamente por las mujeres y sus consecuencias para el pleno acceso de éstas a la ciudadanía. Se trabaja desde la perspectiva de la historia de las mujeres y de las relaciones de género, así como de los debates historiográficos sobre memoria e historia oral; utilizando amplia bibliografía de referencia sobre los feminismos del período, la dictadura y la apertura democrática. De este modo, se pretende pensar la historia del debate acerca del trabajo doméstico, encabezado por feministas de esa generación, como un punto crucial para las mujeres en nuevas perspectivas democráticas que se construyeron en el ámbito político brasileño, en la segunda mitad de los años 1980.

Palabras clave: Trabajo Doméstico, Historia de los Feminismos, Democracia, Memoria, Brasil.

\section{Abstract:}

This article utilizes as sources memories of women who were feminist militants during the military dictatorship in Brazil and who also participated in the political opening in the country from the mid-1980s. In these memories are investigated, from the point of view of these women, who were politically engaged in feminisms and often in the left, the interlocutions between feminist problematizations about domestic work and the perspectives of a democratic society that begins to be unveiled in those years. Under the slogan "democracy in the country and at home", feminist debates articulated public and private by denouncing the domestic workload that was carried out almost exclusively by women and its consequences for full access to citizenship. This work uses interviews conducted under a broad project coordinated by Professor Cristina ScheibeWollf at the Federal University of Santa Catarina in Florianópolis, Brazil, funded by CAPES (Coordenação de Aperfeiçoamento de Pessoal do Nível Superior), entitled "Mulheres de luta: feminismo e esquerdas no Brasil (1964-1985)", of which this author is a member. The article is based on the perspective of women's history and gender relations, as well as the historiographic debates on memory and oral history, crossing a wide bibliography of reference on the feminisms of the period, dictatorship and democratic opening. In this way, the intention is to think about the history of the debate on domestic work headed by feminists of this generation as a focal point for women in the new democratic perspectives that were built in the Brazilian political sphere in the second half of the 1980s.

KEYWORDS: Domestic Work, History of Feminisms, Democracy, Memory, Brazil.

\section{INTRODUCCIÓN}

Al observar la historia de los feminismos que emergieron en Brasil durante el período de la dictadura militar (1964-1985), los debates acerca del trabajo doméstico (remunerado y principalmente no remunerado) de las mujeres como una reivindicación democrática pueden ser señalados como un recorte no tradicional para pensar la memoria sobre la dictadura brasileña.

Cabe destacar que tanto las discusiones sobre el trabajo doméstico no remunerado, como sobre el remunerado, fueron objeto de investigaciones anteriores (Mello, 2011; Mello, 2016). Para poder 
diferenciarlo, fue seleccionada la terminología "servicio doméstico" haciendo referencia al trabajo doméstico remunerado, de acuerdo con la diferenciación de términos realizada por Mirta Henault (2001), en su libro De la rueca a la red. La economía sumergida. Esta autora, que fue integrante de movimientos feministas desde la década de 1970 en Argentina, ofrece su aporte teórico, junto con la brasileña Cristina Bruschini $(1996,2007)$, en lo referente a las problematizaciones que permean el trabajo doméstico de las mujeres. Como referente importante sobre esa cuestión, cabe citar también a María Ángeles Durán (1983), economista española que publicó su libro $A$ dona de casa: crítica política da economia doméstica, en Brasil.

Gran parte de los problemas relacionados al empleoy servicio doméstico son tratados por estas autoras como las relaciones de producción-reproducción del trabajo, la designación social de la responsabilidad de ese trabajo a las mujeres, la división sexual del trabajo en el hogar, las reivindicaciones por la socialización del trabajo doméstico, el papel del trabajo doméstico gratuito como base de sustento para el sistema capitalista, las relaciones con las nuevas tecnologías en el hogar, etc.

La publicación Necesarias, invisibles, discriminadas. Las trabajadoras del servicio doméstico en el Paraguay (Bareiro, Soto y Valiente, 2005), aporta además el concepto de empleo doméstico de una manera pertinente, relacionándolo con el trabajo doméstico gratuito, señalando que "empleo doméstico" se refiere a cuando alguien es contratada para realizar el trabajo doméstico, que es el mismo que el de la ama de casa. Si bien este régimen de contratación cambia efectivamente las relacionescon el trabajo, no necesariamente interfiere en las tareas desempeñadas.

Estas son actividades que pesan sobremanera en la vida de las mujeres y su relación en este artículo con el acceso pleno de ellas a la ciudadanía, dentro de un contexto de debate y lucha por la democracia. Bajo esta perspectiva, son objeto del presente análisis memorias de feministas que vivieron ese período y estuvieron ligadas, de maneras muy distintas, a la lucha contra la dictadura ya la construcción de la apertura política; así como las interlocuciones entre las problematizaciones feministas acerca del trabajo doméstico y la perspectiva de sociedad democrática que se abre durante ese período.

Además, el compromiso de esas mujeres en la lucha contra la dictadura, por la democracia y los derechos de las mujeres en el recorte temporal propuesto en este artículo, también se articulan aquí como base del análisis. Bajo el lema "democracia en el país y en la casa", los debates feministas ensamblaron lo público y lo privado al denunciar la carga de trabajo doméstico que era ejercida casi exclusivamente por las mujeres y sus consecuencias para el pleno acceso de éstas a la ciudadanía.

Como aporte teórico, además de la bibliografía citada durante el artículo, es importante destacar nombres como el de Helena Hirata (2004) y el de ésta y Danièle Kergoat (Hirata y Kergoat 2003; 2007), la ya citada María Cristina Bruschini (2008), Dominique Fougeyrollas-Schwebel (2009) y, además, la contribución de una de las feministas entrevistadas, Hildete Pereira de Melo (2010). Esta última autora viene históricamente, desde la década de 1980, debatiendo y publicando en Brasil sobre cuestiones referentes al trabajo doméstico dentro de la perspectiva de la teoría feminista. Las obras citadas aquí son apenas algunos ejemplos, teniendo en cuenta que la producción de estas intelectuales en ese campo es bastante amplia.

La propuesta se presenta, entonces, bajo la perspectiva de la historia de las mujeres y de las relaciones de género, así como de los debates historiográficos sobre memoria e historia oral, incorporando bibliografía de referencia sobre los feminismos del período, la dictadura y la apertura democrática. De este modo, se pretende pensar la historia del debate acerca del trabajo doméstico encabezado por feministas de esta generación, como un punto crucial para las mujeres en las nuevas perspectivas democráticas que se construyeron en el ámbito político brasileño en la segunda mitad de los años 1980.

\section{Feminismos y movimientos de MUjeres desde los aÑos 1960 en BrasiL}

Ante la efervescencia política brasileña repleta de nuevas ideas progresistas, y acompañada por una modernización e industrialización creciente desde la década de 1950 (Mello y Nováis, 1998), se desencadena, 
el $1^{\circ}$ de abril de 1964, el golpe cívico-militar en el país, que puso fin al gobierno democrático del entonces presidente João Goulart. Después del mismo, Brasil recién volverá a tener un presidente civil, electo indirectamente, en 1985. Apesar de las especificidades de la dictadura brasileña, las motivaciones del ataque militar al gobierno democrático fueron semejantes a aquellas ocurridas en otros países del Cono Surdurante este período (Coggiola, 2001). Estas estuvieron marcadas por las disputas del mundo bipolar, la intervención estadounidense en América Latina y el crecimiento o fortalecimiento del conservadurismo en sectores de la población civil (Fico, 2004; Ferreira y Gomes, 2014; Napolitano, 2014).

Es en este escenario, de ataque a las libertades democráticas y de terrorismo de Estado, que emergieron y se establecieron en Brasil movilizaciones feministas que son, agrandes rasgos, clasificadas por teóricas e historiadoras como de la Segunda Ola (Hemmings, 2009; Pedro, 2006; Pedro, 2011). Este surgimiento de los feminismos, en este contexto de represión política, puede parecer paradójico, sin embargo, justamente la paradoja sería una característica fundamental de los feminismos, ya indicada en otros sentidos por Joan W. Scott (2002 y 2005). Además, es también característica de la "modernización conservadora” (Duarte, 2005; Goldberg, 1987), que incorpora nuevas prácticas mientras evita transformaciones profundas, algo observable en lo concerniente a las costumbres y, en especial, a las mujeres, en este período en Brasil.

En este contexto, se hace necesario mencionar que existe una amplia bibliografía que busca explicar cómo fue que, en un escenario de persecución a las izquierdas, las movilizaciones de mujeres, no entendidas como "tan políticas" o "tan peligrosas" (como aquellas asociadas al comunismo o socialismo), pudieron existir a pesar del estado de excepción y del terrorismo de Estado. El Año Internacional de la Mujer, establecido como tal por la Organización de las Naciones Unidas (ONU) en 1975, motivó, según Cynthia Andersen Sarti (2004), la ocupación del espacio público, la aparición pública de movimientos y participaciones políticas feministas que trabajaban hasta ese momento en los bastidores de la clandestinidad. De ese modo, si ya existían antes de 1975 grupos de reflexión de mujeres o feministas, así como publicaciones y traducciones de determinadas obras y algunas mujeres vanguardistas debatiendo la "condición femenina"; los agrupamientos de mujeres denominados feministas aprovecharon el aval de la ONU como oportunidad para llevar esos debates al espacio público (Wolff, Crescêncio y Pedro, 2016).

Esa relación con la ONU integra un discurso fundador de los feminismos de esa generaciónen Brasil, que está estrechamente ligado ala historia del desarrollo de los centros urbanos e intelectuales del país, principalmente el eje Rio-São Paulo. A pesar de que otras historias más periféricas, plurales y diversas de los feminismos, principalmente de los movimientos de mujeres, también son contadas, esa historia, considerada más hegemónica, no dejade existir, ni de ser retransmitida por muchas mujeres que participaron de la misma y que hoy la rememoran y escriben, a partir de los lugares de importancia que ocupan en las principales universidades del país. En resumen, el hecho de evocar esta característica sirve para remarcar que esta narrativa no es la única presente en la historia de los feminismos de ese período sino que se constituye como un "hito fundador" que interesa en este artículo, ya que las tres protagonistas cuyas memorias serán observadas aquí, forman de alguna manera, aun partiendo de perspectivas muy distintas entre sí, parte de esa historia más hegemónica de los feminismos brasileños.

\section{Consideraciones Metodológicas}

Este artículo es el primer resultado de la participación en el proyecto de investigación "Mulheres de luta: feminismo e esquerdas no Brasil (1964-1985)", coordinado por la profesora Cristina Scheibe Wolff, en el Departamento de Historia de Universidad Federal de Santa Catarina (UFSC) y con vigencia entre 2017 y 2019. Este proyecto fue contemplado por la convocatoria 12/2015 CAPES (Coordinación de Perfeccionamiento de Personal del Nivel Superior), del Programa "Memórias Brasileiras - Conflitos Sociais", Proceso 88887.130836/2016-00. Tiene como objetivo analizar el feminismo, percibiendo la especificidad de su constitución como movimiento social y conjunto de ideas en Brasil, en el período de la dictadura 
militar. Además de contemplar sus interfaces con las organizaciones y los movimientos de enfrentamiento de la dictadura: partidos, organizaciones armadas, movimientos de familiares de presxs y desaparecidxs, grupos de exiliadxs, entre otros movimientos. La principal problemática de este proyecto es que el feminismo ha planteado un conflicto social importante en la sociedad brasileña, que se consolidó especialmente en su interacción con grupos de izquierda durante el período de la dictadura (1964-1985) y que, durante este período, trajo cambios sociales significativos. Las fuentes de investigación son variadas: documentos de archivo, producción bibliográfica de las mujeres protagonistas de esa historia (del período estudiado y también contemporánea), así como también entrevistas realizadas aalgunas de esas mujeres (Wolff, 2015).

Uno de los objetivos del proyecto es la construcción de un webdoc sobre la temática, una especie de libro virtual con fines didácticos, disponible gratuitamente en una página en internet, en el cual se pretende colocar a disposición textos, documentos y entrevistas separadas y editadas conforme a temáticas que son entendidas, a través de nuestras investigaciones, como importantes para los feminismos de aquella generación. Entre esos temas se encuentra contemplada la cuestión del trabajo doméstico.

Este tema fue bastante abordado por los feminismos de matriz materialista que se fortalecieron en Brasil en el período en diálogo con las izquierdas y que identificaron la doble o triple jornada de trabajo a través de cálculos del importe de trabajo no pago que las mujeres prestaban a la sociedad. Además de este análisis materialista, el trabajo doméstico fue también abordado dentro de la percepción de sororidad, hermandad de opresión específica de las mujeres. Estos análisis sugieren que estas cuestiones, que eran entendidas como comunes a todas las mujeres, influenciaron fuertemente las primeras generaciones de los feminismos de la Segunda Ola de Occidente (Mello, 2007, 2011, 2016).

De este modo, el trabajo doméstico se destaca en los discursos feministas de la década de 1970 en Brasil y persiste, a pesar de los avances, como un problema no resuelto por las movilizaciones feministas. En la actualidad, se calcula a través de la Pesquisa Nacional por Muestra de Domicilios (PNAD) del Instituto Brasileño de Geografía y Estadística (IBGE) (Brasil, 2018), que las mujeres aún ejecutan el doble de horas semanales que los hombres en tareas domésticas, a pesar de su amplia inserción en el mercado formal de trabajo (casi 83\% del total de mujeres) (Silveira, 2017). Se destaca, en este sentido, la importancia que tiene este debate en la actualidad.

Cabe aclarar que las entrevistas aquí utilizadas fueron elaboradas para la investigación del proyecto "Mulheres de Luta", y por lo tanto, no poseen cuestiones específicas sobre el trabajo doméstico. Las preguntas giraron en torno a las relaciones entre feminismos e izquierdas y de las identificaciones y contactos de las entrevistadas con el feminismo. Al realizar las entrevistas y debido a intereses de la investigación, especialmente en el caso de Hildete Pereira de Melo y de Luci Teresinha Choinacki, cuya historia de actuación y su sensibilidad con la temática eran conocidas, sí les fue consultado sobre la temática del trabajo doméstico. Es importante destacar esta característica ya que, a pesar de que las otras entrevistadas (en el marco del proyecto "Mulheres de Luta" fueron realizadas hasta el momento un total de veintidós entrevistas), probablemente tienen posicionamientos y opiniones sobre el trabajo doméstico y los feminismos, no fueron indagadas directamente para hablar sobre esa temática durante las entrevistas lo que produjo que fueran surgiendo otros temas como prioritarios.

Por citar un ejemplo, la pregunta número 8 de la entrevista se refiere a cuáles son los temas que eran discutidos y las propias entrevistadas desarrollaban temáticas sobre las que se indagaba. Si se acuerda con la percepción de que se recuerda y se escribe la historia a partir de inquietudes del presente (Bloch, 2001; Delgado y Ferreira, 2014; Freitas, Araújo y Sales, 2017; Pollack, 1989), fue posible percibir, también, que muchas de las temáticas señaladas fueron aquellas con las cuales las entrevistadas se identificaron por muchos años o que interpretaron como notables para la historia de aquel período a partir de una mirada desde el presente (o más próxima del presente, ya que las entrevistas fueron realizadas en 2017). Lo que significa que, teniendo en cuenta la metodología de la historia oral, se puede pensar que las memorias y los testimonios, fuentes del presente artículo, son siempre situadas, con lo cual pueden aportar más indicios y cuestiones sobre 
el período en que los testimonios fueron recolectados que necesariamente sobre el período que fue tema de la entrevista.

Las tres entrevistas fueron realizadas durante el transcurso del año 2017 y una breve biografía de las entrevistadas será presentada a continuación.

Fani Miranda Tabak, nació en 1972, es profesora de literatura de la Universida de Federal do Triângulo Mineiro (UFTM), especializada en estudios de narrativa poética e historiografía, modernidad y estudios de género. Miembro del Grupo de Trabajo "La Mujer en la Literatura" de la Asociación Nacional de PosGraduación e Investigación en Letras y Lingüística (ANPOLL), que existe desde 1984. Tiene interés por el área de historiografía, participación y autoría de mujeres e independencia en América Latina, principalmente en un corpus no canónico.

Fani es nieta de Fanny Tabak, socióloga carioca, considerada fundadora del primer Núcleo de Estudios sobre la Mujer en una universidad brasileña (PUC-Rio), al final de la década de 1980. Fanny se había afiliado al PCB (Partido Comunista Brasileño) y tuvo una destacada actuación en el movimiento de mujeres en la década de 1940, participando de la fundación de la Federación de Mujeres de Brasil. Fue también una de las militantes señaladas como pionera de los feminismos brasileños de las décadas de 1970 y 1980, dedicándose por muchos años a estudiar y militar en las cuestiones referentes a la participación de las mujeres en la ciencia y en la política (Vasconcellos y Lima, 2016). La entrevista discurrió sobre feminismo inter-generacional y las percepciones de la entrevistada, como feminista, de esos debates en el tiempo, en su vida y, también, en la de su abuela. Los abordajes sobre la temática del trabajo doméstico son bastante puntuales, pero aún así, como podrá observarse más adelante, enriquecen cualitativamente los argumentos sobre este tema.

Hildete Pereira de Melo, nació en 1943, es economista y, desde 1972, profesora de la Universidad Federal Fluminense (UFF). Tuvo participación en grupos de resistencia a la dictadura. Fue directora financiera del Centro Internacional "Celso Furtado" de Políticas para el Desarrollo, entre 2004 y 2007; actuó en la Secretaría de Políticas para las Mujeres (SPM) de la Presidencia de la República de Brasil, entre 2009 y 2010; y posteriormente, entre 2012 y 2014. Tuvo una actuación feminista destacada en la academia y en la política institucional y estuvo comprometida con muchos grupos y diferentes frentes de militancia de las mujeres, incluyendo los debates de la Asamblea Nacional Constituyente, de 1987. Actualmente, es uno de los principales nombres de Brasil en el campo de la producción científica sobre el trabajo de las mujeres, con énfasis en las temáticas del trabajo doméstico, no remunerado y de cuidado (Melo y Bandeira, 2013; Melo y Castilho, 2009; Melo, Considera y Sabbato, 2013). Su entrevista es una de las más extensas de las realizadas para el proyecto de investigación "Mulheres de Luta: feminismo e esquerdas no Brasil (1964-1985)", con la salvedad, como en los otros casos, de que esta entrevista no está enfocada específicamente en la cuestión del trabajo doméstico.

El discurso de Pereira de Melo es un discurso de quien está acostumbrada a contar repetidas veces esa historia, una historia de los feminismos brasileños que atraviesa su propia constitución como intelectual. Además, es un testimonio permeado por reflexiones académicas feministas de alguien que produce científicamente sobre el trabajo de las mujeres. En este sentido, debe resaltarse que los tres testimonios presentados en este artículo están permeados por la militancia, formación y vivencia feministas, y eso ciertamente constituye sus discursos. Sin embargo, el hecho de que Hildete piensa y produce teóricamente, desde hace muchos años, sobre el trabajo doméstico remunerado y no remunerado de las mujeres en Brasil, es una cuestión que precisa ser destacada en este análisis.

Luci Teresinha Choinacki, nació en 1954, es agricultora y militante a favor de la agricultura orgánica y familiar. Hija de agricultores, comenzó a actuar en las Comunidades Eclesiásticas de Base (CEB) y en movimientos sociales. Ingresó al Partido de los Trabajadores (PT) en 1982 y participó de la formación de la Central Única de los Trabajadores (CUT), de la construcción del Movimiento de Mujeres Campesinas (MMC) y del Movimiento de los Trabajadores Rurales Sin Tierra (MST) de Santa Catarina. Fue la única mujer electa, en 1986, para la Asamblea Legislativa del Estado de Santa Catarina y fue diputada estatal 
constituyente, en 1987. En 1990, fue elegida para la Cámara de Diputados, siendo reelegida para los mandatos de 1999, 2003 y 2011. Tuvo una actuación central en los debates sobre la jubilación para trabajadores rurales, en especial para las mujeres, en la Constitución de 1988 (Medeiros, 2017) y es autora del Proyecto de Ley de 2005 (Revista gênero, 2006), que reguló la jubilación de las amas de casa de bajos ingresos: el sistema especial de inclusión de seguridad social para trabajadores y trabajadoras sin ingreso propio que se dedican exclusivamente al trabajo doméstico de su hogar.

A diferencia de las entrevistadas citadas anteriormente, cuyas entrevistas fueron realizadas en una sala en la universidad, durante el "13 Mundos de Mujeres e Fazendo Gênero 11" en Florianópolis, en 2017, Choinacki fue entrevistada, a petición suya, en su huerta orgánica en el barrio de Rio Vermelho en Florianópolis. Allí es donde trabaja actualmente, todos los días de la semana. Esta característica, así como su propia historia de vida, que difiere del perfil académico/institucional de Tabak y Pereira de Melo, puede ser captada a lo largo de su entrevista, ya que trae puntos de articulación importantes entre la militancia política de base y la academia, como será señalado más adelante.

Las tres entrevistas presentes expresan una variación en los perfiles de las entrevistadas, en lo referente a la pertenencia generacional, la actuación intelectual y política, las visiones de mundo y las perspectivas del feminismo. Esta variación es importante para pensar sobre la amplitud que alcanza el debate sobre el trabajo no pago de las mujeres, el trabajo familiar y el trabajo doméstico dentro de los feminismos y en un recorte temporal extendido.

\section{El trabajo doméSTico como CUESTIÓN FEMinista}

Además de la historia de los feminismos desde los años 1960, y principalmente 1970, y el contexto en el cual las entrevistas aquí utilizadas fueron producidas, es crucial - para la justificación de este análisis-pensar en la importancia de los debates acerca del trabajo doméstico para aquella generación e, inclusive, para la actual. En investigaciones anteriores, fueron abordadas las discusiones feministas de la Segunda Ola en Brasil y en el Cono Sur sobre el trabajo doméstico (Mello, 2011), a través de la producción impresa de los feminismos: periódicos, panfletos, disertaciones, tesis, artículos y libros. Posteriormente, fue desarrollado el debate feminista sobre el trabajo doméstico en la gran prensa comercial brasileña (Mello, 2016), teniendo en cuenta el mismo recorte temporal que las investigaciones anteriores, entre las décadas de 1970 y 1980 . Estas investigaciones posibilitaron la inserción en las discusiones acerca del trabajo doméstico, principalmente el no remunerado, y la articulación y centralidad en las reivindicaciones feministas, con las características de los propios movimientos en aquellos años, en especial la búsqueda por cuestiones generales que afectaban a todas las mujeres.

Un punto notable, principalmente en los años 1970, fue la percepción de las mujeres que se identificaban públicamente como feministas, en relación a que el problema del trabajo doméstico era un problema de las mujeres como categoría, un problema colectivo y no de algunas de manera individual. La principal referencia son los años 1970, ya que puede observarse en las producciones escritas de los feminismos, a partir de la década de 1980 como la temática del trabajo doméstico y no remunerado de las mujeres pierde espacio en relación a otros debates, como violencia, sexualidad, salud y derechos reproductivos. Tal percepción es corroborada por el testimonio de Pereira de Melo. Cabe destacar que se está haciendo uso aquí de la memoria de la entrevistada para, de hecho, corroborar lo que fuera percibido en más de una década de investigación de archivo de la autora de este artículo (Mello, 2016). Comenta sobre el tema lo siguiente:

Quienes no eran del área del trabajo no tenían mucha sensibilidad, entonces esa discusión del trabajo no pago, era aún embrionaria en nuestro medio, y acabábamos siendo engullidas. Por ejemplo, porque en ese momento explotala cuestión de la violencia y la grieta de los grupos feministas sobre SOS Corpo ${ }^{1}$ (...) Habían ocurrido crímenes: es la época de la serie 'Quien ama no mata', que es una frase feminista. Fueron las feministas de Belo Horizonte que inventaron 'quien ama no mata', cuando fue el asesinato ${ }^{2}$ de Eloísa Ballesteros. ${ }^{3}$ 
Al contar esa historia, que prioriza determinadas temáticas y la superación de otras, se relata la historia de un feminismo hegemónico. No se debe pensar de manera engañosa que, por ser hegemónico, esos grupos hayan enfrentado pocas dificultades para establecerse, o que las militantes, inclusive las académicas, no hayan enfrentado profundos desafíos en todas las esferas de sus vidas debido a su posicionamiento político como feministas. Pereira de Melo (2017) también aborda esta cuestión en su entrevista (2017), explicando que ni su disertación de maestría ni su tesis de doctorado hablaban de mujeres o tenían temáticas feministas, debido a la dificultad de debatir esas cuestiones en los departamentos de Economía en Brasil en las décadas de 1970 y 1980. Ella afirma que "no osó" ir en esa dirección, a pesar de tener durante ese período una pronunciada militancia feminista. Su disertación data de 1979 y su tesis de 1993.

Estas son historias de mujeres intelectuales, en gran parte de estratos medios, en una gran mayoría heterosexuales, que rompieron barreras al ocupar espacios tradicionalmente masculinos en la esfera pública, mientras estaban también casadas o divorciadas. Madres de familia desempeñando, si no todas, muchas de las actividades que eran esperadas de las madres de familia de estratos mediosde aquellageneración. ¿Cuál era el significado de esto?

En gran medida, significaba que no desempeñaban ellas mismas gran parte del trabajo doméstico para sus familias, como ocurría con la mayoría de las amas de casa y sucede aún en nuestros días, pero que tenían responsabilidad sobre aquello que, por estar ocupadas con profesiones, carrera y militancia, no podían desempeñar. Entonces, les cabía delegar esas tareas a otras mujeres, de condición social más precarizada que la de ellas. Las empleadas domésticas --que desempeñaban también las funciones de niñera, ama de casa y cocinera-- trabajaron y aún trabajan para las familias de muchas feministas brasileñas. A pesar de la responsabilidad por gestionar ese trabajo, encontrar y contratar a alguien que lo lleve a cabo, e inclusive realizar esas funciones en ausencia de la persona contratada (por regla general, estas actividades recaen sobre esas madres de familia feministas), existía esa diferenciación entre quien de hecho realizaba el trabajo doméstico y las amas de casa que contaban con empleadas (Moraes, 1985, 1990).

Es importante reiterar que se está aquí reproduciendo, de alguna manera, una historia de los feminismos que puede ser entendida como hegemónica, ya que esla historia repetida en fuentes de investigación, entrevistas y referencias bibliográficas, producidas por muchas de esas feministas de estratos medios, protagonistas de esos acontecimientos en grandes centros urbanos. Por lo que estos datos indican, ese era el perfil de las mujeres que participaban en grupos feministas brasileños en aquel período. Pero esa historia, como siempre en la ciencia histórica, aún puede ser disputada por otros datos y fuentes, tal vez por la historia de grupos feministas aún no estudiados y/o de perfiles diferentes. De cualquier manera, son historias de articulación y resistencia, de ruptura de barreras establecidas para las mujeres y que sufrenla autocrítica de sus límites por parte de las propias entrevistadas.

Al considerar este perfil, existen innumerables factores a ser examinados sobre esa cuestión: la dificultad de llevar el discurso de lo "personal es político" a las relaciones familiares en lo referido a la división de tareas y división del trabajo doméstico entre los miembros de la familia; la profunda desigualdad de ingreso latino-americana que permitía y permite que, aún siendo familias de estratos medios o medios-bajos, puedan contratar empleadas, aunque sea por jornada; y una cierta vergüenza de las feministas de estratos medios, de esa generación, por entender que su inserción en la esfera pública solo fue posible debido a una profunda desigualdad y a la explotación del trabajo de mujeres que vivían en situaciones más vulnerables que ellas, como es posible observar en el discurso de Pereira de Melo:

Nosotras éramos beneficiadas por él [el trabajo doméstico]. Todo el mundo tenía empleada, quiero decir, la podían tratar bien, pagar lo justo, pero nosotras somos fruto de esa sociedad en la cual participar de la política lleva tiempo, toma tiempo salir de casa. Entonces era el retroceso que permaneció.

Estos son factores poco discutidos por las feministas del período (Mello, 2011), que estaban acostumbradas a compararse con las mujeres de las clases trabajadoras, entendiéndose como "menos oprimidas" (Nós 
Mulheres, 1976, p.2). Posición que también necesita ser articulada con la fuerza de las teorías marxistas en los feminismos de esa generación en Brasil y su posible resistencia a un debate norteamericano, como el de Betty Friedan (1971), que podría ser asociado al imperialismo y a la dictadura. De ese modo, no se profundizaron los debates sobre la división de tareas en los hogares de estratos medios (Silva, 1979) o nociones como la de "maternidad transferida", acuñada por una de las feministas de esa generación, Suely Gomes Costa (2002), años más tarde. Lo que no quiere decir que la cuestión no haya sido abordada. Como la misma Pereira de Melo señala:

Escribí la historia de ellas, de Rio, de las asociaciones, era Nair Jane, que es un monumento a la empleada doméstica, que está luchando hasta hoy, es la presidenta del Sindicato de Nova Iguaçu. Y después no hice nada más. Comencé a trabajar nuevamente sobre el tema de la empleada doméstica en la década del 90, cuando comencé a trabajar mucho con el mercado de trabajo, en una investigación del IPEA [Instituto de Investigación Económica Aplicada]. (...) Fue en el '96 eso, me sorprendió el éxito. A ese artículo, Estudos Feministas lo publicó en el '98, el artículo (...) ya desmenuzando la PNAD y tal. A partir de ese momento entró en la agenda (...) diría que antes había estudios, así, más puntuales, con una mirada sociológica, pero no con números para mostrar que la ocupación de las domésticas es la principal ocupación de las mujeres brasileñas.

Además del material encontrado en los tres periódicos feministas brasileños más citados de los años 1970 y1980 (Brasil Mulher-1975-1980, Nós Mulheres- 1976-1978 y Mulherio-1981-1988), así como material bibliográfico (Beauvoir, 1970; Friedan, 1971; Prado, 1979) que circulaban por el país, la temática trabajo tenía un espacio cautivo en esos feminismos tan atravesados por el marxismo y organizados en el seno de la lucha contra la dictadura. Y en ese espacio, era posible debatir sobre el trabajo no remunerado, aunque fuese muchas veces visto como un "problema de las otras", y no de ellas mismas, de las feministas con vida profesional y de amplia actuación en la esfera pública Pereira de Melo comenta:

Entonces, esa cuestión no era una cuestión trabajada. (...) Antes de ser fundado el NEIM [Núcleo de Estudios Interdisciplinares sobre la Mujer], en 1983, allá en Bahía, Fanny Tabak creó el Núcleo de Estudos da Mulher en la PUC [Pontifícia Universidad Católica] que frecuenté mucho. La primera vez que hablé, no dar clases, que hablé en público, fue en una conferencia convocada por el núcleo de la PUC, junto con el Departamento de Ciencias Sociales de la PUC, sobre mujer en el mercado de trabajo. No fui yo la invitada, sino Branca [Moreira Alves], pero Branca dijo: Hildete, para hablar de trabajosos vos, no soy yo. ¡Tenés que ir vos! Y yo dije: ¡ay dios mío!, ¿ Cómo voy a hablar yo? ¡Nunca hablé en público! Una platea de 150 personas que no eran lxs alumnxs de economía, con quien estaba acostumbrada a hablar, iy ahí hable sobre la invisibilidad del trabajo de la mujer! Me acuerdo hasta el día de hoy. Después, escribí un artículo para el Diario de los Economistas sobre la invisibilidad del trabajo femenino. ¡Del trabajo no pago! (risas). Estábamos abriendo esa discusión, porque esa es una discusión que aparecía cuando se trabajaba igual salario, o existe un problema de desigualdad del salario, pero también cuando hay sobrecarga de otro trabajo. Entonces, fue la primera vez, porque eneconomía...

- ¿Enqué año fue ese artículo?

- 78. (...)

Pereira de Melo recuerda la permanencia, debidamente compaginada, y no una permanencia anacrónica, sino una permanencia, de prácticas, debates, pautas y de la propia agenda feminista. Esta asociación se realiza a partir de décadas de compromiso con la militancia feminista brasileña, acompañando las disputas y la recuperación de pautas.

Las discusiones, hoy cuando veo el movimiento feminista hablando, digo: ¡ay, volvió todo! ¡La pauta no cambia! (risas). Es interesante observar, porque la juventud de hoy piensa que está realizando una gran novedad. Ellas cambiaron un poco los adjetivos y los conceptos, pero en el fondo es la misma cuestión. Por ejemplo, estoy en la PartidA, con el grupo allá en Rio, y la discusión, y me quedo mirando y es genial, porque parece que estoy viviendo 78, 77, en el Centro de la Mujer Brasileña.

En efecto, como señala Tiburi (2016), "recordemos que el movimiento feminista \#partidA, surgió en 2015 cuando algunas feministas discutieron la posibilidad y el sentido de la creación de un partido feminista en Brasil”. Pereira de Melo rescata la dinámica de \# partidA, la reconoce como propia de sus propias experiencias previas. 
Y no hay coordinación, y tiene coordinación, quien manda, quien no manda, ¿̨cuál es la voz? Mirá, es parecido. Claro que no es lo mismo, porque quien vivió esa época no son ustedes que viven la actualidad, pero esa es la trayectoria.

Por su parte, la entrevista concedida por Choinacki trae un contrapunto muy importante. A pesar de haber ocupado cargos políticos de relevancia como diputada provincial y federal, Choinacki tiene una historia de vida muy diferente a la mayor parte de las brasileñas que encabezaron los grupos feministas más conocidos de la década de 1970. Inclusive, porque su militancia data de la década de 1980, y los resultados de esa militancia guardan relación con la apertura política brasileña, recordando que la Carta Constitucional es de 1988. Agricultora, tenía el deseo de ser profesora, pero no pudo continuar sus estudios ya que por ser hija primogénita de siete hermanxs, tuvo que ayudar a criarlxs. Es madre de cuatro hijxs, uno de ellos con discapacidad y con necesidad de cuidados específicos. Ingresó en la vida política a través de los movimientos sociales ligados a la Teología de la Liberación, a la Pastoral de la Tierra, a través de la cual se involucra en el Movimiento de Trabajadores Rurales Sin Tierra (MST) y en el Movimiento de Mujeres Campesinas (MMC) de Santa Catarina.

Esas cuestiones se reflejan en su actuación política. Choinacki obtuvo importantes resultados de la militancia dentro de sus gestiones, principalmenteen lo que se refiere a derechos laborales antes negados a las trabajadoras rurales (como la licencia por maternidad o jubilación); jubilación para amas de casa de bajos ingresos (una población que antes de esta conquista no tenía acceso a la seguridad social, totalmente dependiente de familiares); debates sobre la feminización de la pobreza y también lo que hoy se denominarían políticas del cuidado. Esos logros son resaltados por ella cuando fue indagada sobre ese período.

Mirá, al comienzo fue así: los grupos feministas (...) tenían un poco de dificultad. Después, se fue abriendo el proceso, para las agricultoras, principalmente, con la cuestión del trabajo doméstico. El trabajo doméstico fue una cosa muy, muy dura para ser debatida. Creamos una manera en que las mujeres amas de casa se jubilarían, ahí apareció una crisis de concepciones. Pero no era: '¡la mujer tiene que quedarse en casa!'; sino reconocer que existía un público, aislado de todo el proceso y que precisaba de un tratamiento diferenciado. Es como una transición. Entonces, tuvimos problemas de inicio.

Es importante recordar que entre mediados de 1980 y durante la década de 1990, se institucionalizan y fortalecen de forma preponderante los estudios feministas y de género en Brasil como campo de producción de conocimiento, cuando se multiplican, con gran esfuerzo los frentes feministas en universidades, las publicaciones, los núcleos de investigación y eventos del área. Es en ese contexto de articulación entre militancia feminista política y académica (para diferenciar los espacios de actuación pero sin desconsiderar el carácter político del feminismo académico) que se lograron las conquistas legales de derechos de las mujeres trabajadoras brasileñas, citadas por Choinacki:

Después, debatimos mucho en todo Brasil, hicimos seminarios, plenarios, ahí un gran grupo de feministas asumió la bandera y fueron en conjunto a realizar la conferencia y discusión: Hildete Pereira, no sé si alguien la conoce; la Doctora Salete, que era una jueza de Rio de Janeiro; aquí del estado, Joana Pedro, y así siguen...Pero así, en todo Brasil, reunimos muchas mujeres después que asumieron la causa. Inclusive realicé una comisión parlamentar, para hacer un trabajo: la pobreza tiene una cara que es el rostro de las mujeres. Porque encualquier crisis, cualquier golpe que hay, quien[es] sufre[n] primero el despido, el castigo, son las mujeres, porque sienten el costo de la alimentación, el cuidado de los hijos, la enfermedad en casa, las personas con discapacidad para cuidar, y digo esto con conocimiento de causa, ¿̨no es así? Entonces', fuimos introduciendo esa discusión y ésta alcanzó una dimensión bien grande. Inclusive, hubo investigaciones: ' ¿Cuánto sería si hubiese que pagar el trabajo doméstico que las mujeres realizan?' (...) El trabajo gratuito que las mujeres están brindando para la sociedad, que no es reconocido de ninguna forma.

Continúa la entrevista comentando la necesidad de aparatos del Estado volcados a la distribución del ingreso y socialización del trabajo de cuidado, entendiendo que ese trabajo recae principalmente sobre las mujeres. Ella trae demandas feministas pero su formación política está mucho más ligada a los movimientos de mujeres.

Otra articulación importante en el discurso de Choinacki se refiere el debate de la división de las tareas en el hogar que parece ser una pauta común en esas esferas. 
Es totalmente diferente, pero en los dos sectores de mujeres, tanto urbano como rural, aún pesa para las mujeres, infelizmente. El trabajo más agotador, diario, que no tiene la contribución de la familia, de los hijos, del marido, de su compañero, sea lo que sea, la participación en el trabajo. Voy a decir, cuando hay participación, las cosas fluyen diferente, nos sentimos mejor, hasta porque si uno cocina y el otro lava, es diferente (...) Y, también, para pedir es tedioso, se pide una vez, dos, después te cansás de estar pidiendo, entonces las personas tienen que asumirlo como una concepción de vida, de valores.

Se puede pensar en la permanencia de esa cuestión cuando se observan datos recientes del IBGE (2018): en 2016, las mujeres brasileñas aun dedicaban 18,1 horas semanales a que haceres domésticos, mientras que los hombres solamente dedicaban 10,5 horas. Las mujeres que aun tenían ingresos medios casi $\mathrm{R} \$ 600$ (reales) menores que los varones, ellas configuraban apenas un 10,5\% (contra una tasa mundial de 23,6\%) de la representación política y ocupaban 39,1\% de los cargos gerenciales, a pesar de su mayor escolaridad (23,5\% contra $20,7 \%$ de ellos con enseñanza superior). Son también la mayoría entre lxs desempleadxs, con un índice de $13,4 \%$, contra $10,5 \%$ entre los varones. Choinacki continúa:

Es, exactamente, de participar, porque si una personasola hace todo, se cansa, se agota. Pero si quien está junto [a ti] participa, cambia, la vida se vuelve diferente. Si tenés que hacerlo sola, vas mirando a la otra persona ahí sentada, cómoda, ¡al poco tiempo te da ganas de revolear la silla para ver si se despega de ahí! (risas).

Esa cuestión lleva a otro aspecto de la vida familiar que está vinculada con los feminismos, que remite a la maternidad y, también, a la vida doméstica. Apesar de que Miranda Tabak, nieta de uno de los más destacados nombres del feminismo brasileño del período, no haya abordado el tema del trabajo doméstico en su entrevista, llama la atención en este contexto, al reflexionar sobre el feminismo inter-generacional, cómo ella observa que la posición de feminista es reconocida y admirada por muchas personas, pero no siempre bien vista en la familia. Hizo referencia a su abuela, y también a símisma, como académica y feminista. Una madre (o abuela) con posición social destacada, generalmente, no puede desempeñar lo que tradicionalmente se espera de una madre que, como ya comentaba Danda Prado (1979), es básicamente, servir a la familia incondicionalmente, con mucho amor.

Pienso que el impacto del feminismo sobre la familia es muy diferente del impacto sobre las personas que se inspiran en vos. Sobre la familia es una cosa más complicada, más delicada. Pienso que vivo un poco esohoyen la práctica con mis hijas. Entonces, a veces, no es fácil para la familia percibir aquello que los otros perciben, que es importante para vos o que te vuelve una persona inspiradora. A veces, eso, para la familia, puede ser lo contrario. (...) Pienso que es difícil -sobretodo- para la mujer. Existe una serie de opiniones sobre la mujer, nadie nunca preguntó lo que ella piensa, de hecho. Pienso que el día en que hable, todo el mundo sale corriendo, pero, por suerte, los hijos no nos preguntan: ¿madre que piensas del hecho de ser madre? Entonces, nos quedamos sólo en las entrelíneas de las cosas, porque es una representación muy difícil.

Estas diferencias de percepciones sobre el trabajo doméstico y las expectativas resultantes depositadas sobre las mujeres, principalmente las que son madres se dan de formas muy variadas desde los años 1960 y 1970 hasta la actualidad. Sin embargo, algunas continuidades pueden ser observadas, y análisis recientes sobre el trabajo doméstico y el trabajo reproductivo realizado por las mujeres señalan ese trabajo como obstáculo al acceso pleno a la ciudadanía. No se cuestiona la importancia del trabajo reproductivo, sino por el contrario, existe una amplia bibliografía disponible, tributaria de los estudios feministas y de género, que percibe un "uso presumible" que el Estado, la economía y la sociedad realizan del trabajo considerado femenino, uso que interfiere directamente en el acceso de las mujeres a los derechos fundamentales (Mariano y Souza, 2015), cuando parte considerable del tiempo y de la energía de ellas es ocupada en tareas de manutención de la colectividad (Abreu, Hirata y Lombardi, 2016).

\section{Algunas CONSIDERACIONES Finales}

A partir de estas observaciones, es posible introducir algunas preguntas. ¿Cuánto ayudan los discursos y las memorias de esas mujeres a pensar la historia de los debates acerca del trabajo doméstico y de los feminismos 
como enfoque del conflicto social en Brasil partiendo de la dictadura militar, pero también en nuestros días? ¿Cuántos de los problemas señalados por ellas en los testimonios presentados, y otros también encontrados en las producciones feministas del pasado, en panfletos, periódicos, grupos de reflexión y palabras de orden en las calles resuenan con profundo significado para las mujeres brasileñas aún en la actualidad? ¿Cuánto pesa cuantitativa y cualitativamente, de diferentes formas, la cuestión del trabajo no pago que las mujeres realizan para sus familias y para la sociedad (del cual nuestro Estado y las empresas son absolutamente dependientes), en las elecciones y opciones de vida de las mujeres brasileñas?

Dentro de la perspectiva del golpe de estado de 1964 y del régimen de excepción en los años que le siguen, estos debates ganan relevancia. A pesar que el golpe de 1964 fue vivido durante la vida adulta y con militancia política solamente por Pereira de Melo; crecer, militar y vivir durante la dictadura y vivenciar la apertura política de los años 1980 fue el telón de fondo de las trayectorias de las tres entrevistadas. Sobre la relación entre feminismos, mujeres y golpe, por ejemplo, es posible observar los esfuerzos del gobierno militar en exaltar la importancia de la familia tradicional para la salud física y mental de la juventud, subrayando la imprescindible figura materna en el hogar (Duarte y Lucas, 2014), lo que evidencia el carácter conservador del régimen y su preocupación con los cambios que venían ocurriendo en el modelo tradicional de familia (Colling, 2015). Estas son cuestiones que ayudan a pensar los avances conservadores en la contemporaneidad, a construir paralelos y reconocer el valor de los debates clásicos al respecto y del camino trazado y consolidado por estas mujeres aún para nuestra generación.

¿Cuántos paralelos es posible construir entre la dictaduray el momento político que estamos viviendo, en el que derechos básicos son vulnerados en nombre del avance de intereses del capital local e internacional y en el que vivimos una situación política que no es dictadura pero tampoco es democracia? Estos son aspectos centrales en el escenario político brasileño contemporáneo y también en la producción feminista, historiográfica e interdisciplinar y para las cuales no existe una respuesta simple. Es por ese motivo que es de suma importancia profundizar nuestras investigaciones, recolectando más testimonios como los fragmentos aquí presentados y pluralizar nuestras perspectivas sobre estos debates.

Específicamente sobre los testimonios exhibidos, cabe reflexionar sobre sus significados en lo referente a las contribuciones de diferentes generaciones de feministas para el debate del trabajo doméstico y de la ciudadanía de las mujeres. ¿Qué significóel marco de esos debates: a) en la agenda académica y política, b) en la legislación, y c) en las relaciones privadas de las dinámicas familiares? Hildete, Luci y Fani posibilitan visitar cada uno de esos ejes del debate a partir de sus entrevistas. Las especificidades generacionales, espaciales, contextuales, localizadas, nos llevan a diferentes imbricaciones de la temática, a una historia que no es escrita linealmente, sino a través de flujos y reflujos, disputas y negociaciones. Al considerar que "lo personal es político", podemos entender mejor cómo estas diferentes perspectivas se integran en un todo, en ese espacio en que las fronteras artificiales modernas de lo público y privado siempre fueron difusas, en el espacio del trabajo de las mujeres.

Antes que intentar eliminar la falta de linealidad de los trabajos de la memoria, nos interesa aquí pensar cómo la actuación de mujeres feministas en diferentes frentes transforma su entorno, al mismo tiempo en que ellas son transformadas por estas relaciones. En este sentido, los debates sobre epistemología feminista (Haraway, 1995; Harding, 1993; Sardenberg, 2002) son de gran importancia. Es a partir de visiones del mundo y de conocimientos feministas que las tres entrevistadas entrelazan sus consideraciones y es, también, a partir de la interconexión compleja que se establece en los distintos modos de transitar el feminismo que se problematiza la cuestión del trabajo doméstico. Si se toma la posición de las entrevistadas como ejemplo, vemos en la articulación entre las llamadas "feministas políticas" y "feministas académicas" el rico frente de actuación de militancia y producción de conocimiento enfocado en el trabajo de las mujeres. 


\section{Agradecimientos}

Además de a la CAPES como agencia de fomento, es de suma importancia agradecer al equipo del proyecto "Mulheres de Luta" y del Laboratório de Estudos de Gênero e História de la UFSC, sin los cuales hubiera sido imposible escribir este artículo. A la profesora Cristina Scheibe Wolff por todo el apoyo y cariño, tan importantes en mi formación; a las becarias ligadas directamente al proyecto en Florianópolis, con quienes trabajé en 2017: Elaine Schmitt, BinahIrê Vieira Marcellino y Luísa Donelles Briggmann; a las estudiantes que transcribieron las entrevistas utilizadas: Luana Balieiro Cosme, Alina Nunes, Valéria Machado y Jeferson Ramos; y a las entrevistadas por la confianza, por concedernos sus entrevistas, contar sus memorias y compartir con nosotras sus historias. Agradezco también a Matilde Quiroga Castellano por la traducción al español y a Adriana María Valobra por la revisión. Una primera versión de este artículo fue presentado en forma de comunicación oral y recibió una mención en la Universidad Nacional de la Plata en las Vo Jornadas CINIG de Estudios de Género y Feminismos y III ${ }^{\circ}$ Congreso Internacional de Identidades en 2018, donde fui acogida de forma excepcional y recibí contribuciones fundamentales para el presente texto. Agradezco, finalmente, al equipo del Centro Interdisciplinario de Investigaciones en Género (CInIG) de la UNLP por las inestimables contribuciones.

\section{REFERENCIAS}

Abreu, A. R. P., Hirata, H. y Lombardi, R. (Orgs.) (2016). Gênero e trabalho no Brasil e na França: perspectivas interseccionais. São Paulo: Boitempo Editorial.

Bareiro, L., Soto, L. y Valiente, H. (2005). Necesarias, invisibles, discriminadas. Las trabajadoras del servicio doméstico en el Paraguay. Lima: OIT/IPEC Sudamérica.

Beauvoir, S. de (1970). O segundo sexo: fatos e mitos. Vol1. Rio de Janeiro: Nova Fronteira.

Bloch, M. (2001). Apologia da História, ou, o ofício de historiador. Rio de Janeiro: Zahar.

Brasil. (2018). Estatísticas de gênero: indicadores sociais das mulheres no Brasil. Nota técnica número 38. Rio de Janeiro: IBGE.

Bruschini, C. (1996). Fazendo as perguntas certas: como tornar visível a contribuição econômica das mulheres para a sociedade? En GT Gênero e trabalho: questões teóricas, II Congresso Latinoamericano de Sociologia do Trabalho, Águas de Lindóia, Brasil.

Bruschini, C. (2007). Trabalho doméstico: inatividade econômica ou trabalho não-remunerado? (pp. 21-58). En C. Araújo, F. Picanço y C. Scalon (Orgs), Novas conciliações e antigas tensões? Gênero, família e trabalho em perspectiva comparada. Bauru: EDUSC.

Bruschini, M. C. A. (2008). Articulação trabalho e familia: familias urbanas de baixa renda e políticas de apoio às trabalhadoras. São Paulo: FCC/DPE.

Coggiola, O. L. A. (2001). Governos militares na América Latina. São Paulo: Contexto.

Colling, A. M. (2015). 50 anos da ditadura no Brasil: questões feministas e de gênero. OPSIS, 15(2), 370-383.

Costa, S. G. (2002). Proteção social, maternidade transferida e lutas pela saúde reprodutiva. Estudos feministas, 10(2), 301-323.

Delgado, L. A. N. y Ferreira, M. M. (Orgs.). (2014). História do tempo presente. Rio de Janeiro: Ed. FGV.

Duarte, A. R. F. (2005). Carmen da Silva: o feminismo na imprensa brasileira. Fortaleza: Expressão Gráfica e Editora.

Duarte, A. R. F. y Lucas, M. R. L. (Orgs.) (2014). As mobilizações do gênero pela ditadura militar brasileira: 1964-1985. Fortaleza: Expressão Gráfica e Editora.

Durán, M. Á. (1983). A dona de casa: crítica política da economia doméstica. Rio de Janeiro: Edições Graal.

Ferreira, J. y Gomes, Â. C. (2014). 1964: o golpe que derrubou um presidente, pôs fim ao regime democrático e instituiu a ditadura no Brasil. Rio de Janeiro: Civilização Brasileira. 
Fico, C. (2004). Além do golpe: versões e controvérsias sobre 1964 e a Ditadura Militar. Rio de Janeiro: Record.

Fougeyrollas-Schwebel, D. (2009). Trabalho doméstico (verbete). En H. Hirata [et al.] (Orgs), Dicionário crítico do feminismo. São Paulo: UNESP.

Freitas, J. L., Araújo, C. S. y Sales, T. B. (2017). “O que essa entrevista está documentando?”: entrevista com a professora Verena Alberti. História oral, 20(2), 237-251.

Friedan, B. (1971). Mistica feminina. Petrópolis: Vozes.

Goldberg, A. (1987). Feminismo e autoritarismo: a metamorfose de uma utopia de liberação em ideologia liberalizante. Dissertação de Maestreado. Universidade Federal de Rio de Janeiro, Rio de Janeiro.

Haraway, D. (1995). Saberes localizados: a questão da ciência para o feminismo e o privilégio da perspectiva parcial. Cadernos Pagu, 0(5), 7-41.

Harding, S. (1993). A instabilidade das categorias analíticas na teoria feminista. Estudos feministas, 1(1), 7-31.

Hemmings, C. (2009). Contando estórias feministas. Estudos Feministas, 17(1), 215-241.

Henault, M. (2001). De la rueca a la red. La economia sumergida. Buenos Aires: Ediciones Corregidor.

Hirata, H. (2004). Trabalho doméstico: uma servidão "voluntária”? En T. Godinho y M. L. da Silveira, Políticas públicas e igualdade de gênero. São Paulo: Prefeitura de São Paulo - Coordenadoria Especial da Mulher.

Hirata, H. y Kergoat, D. (2003). A divisão sexual do trabalho revisitada (pp. 111-124). Em M. Maruani y H. Hirata (Orgs.), As novas fronteiras da desigualdade: homens e mulheres no mercado de trabalho. São Paulo: Senac.

Hirata, H. y Kergoat, D. (2007). Novas configurações da divisão sexual do trabalho. Cadernos de Pesquisa, 37(132), 595-609.

Mariano, S. A. y Souza, M. F. (2015). Conciliação e tensões entre trabalho e família para mulheres titulares do Programa Bolsa Família. Revista brasileira de ciênciapolitica, 18, 147-177.

Medeiros, S. (2017). Aposentadoria leva autonomia e independência às mulheres do campo. Catarinas. Recuperado de http://catarinas.info/aposentadoria-leva-autonomia-e-independencia-mulheres-campo

Mello, J. M. C. y Novais, F. (1998). Capitalismo tardio e sociabilidade moderna (pp. 559-658). Em L. M. Schwarcz (Org.), História da vida privada no Brasil: contrastes da intimidade contemporânea. São Paulo: Companhia das Letras.

Mello, S. C. (2007). O trabalho doméstico em Nós Mulheres- Trabajo de graduação em Historia. Universidade Federal de Santa Catarina, Florianópolis.

Mello, S. C. (2011). Trabalho doméstico: coisa de mulher? Debates feministas no Cone Sul (1970-1989). Rio de Janeiro: Multifoco.

Mello, S. C. (2016). Discussões feministas na imprensa para mulheres:revista Claudia e o trabalho doméstico (1970-1989). Tesis de Doutorado em Historia. Universidade Federal de Santa Catarina, Florianópolis.

Melo, H. P. (2010). A perspectiva feminista e os trabalhos sobre "Usos do Tempo". Econômica, 12, 6-10.

Melo, H. P. y Bandeira, L. (2013). A Divisão sexual do Trabalho: trabalho doméstico remunerado e a sociabilidade das relações familiares. Gênero, 13, 31-48.

Melo, H. P. y Castilho, M. (2009). Trabalho reprodutivo no Brasil: quem faz? Revista de economia contemporânea, 13, 135-158.

Melo, H. P., Considera, C. M. y Sabbato, A. (2013). 10 anos de mensuração dos afazeres domésticos no Brasil. En 35 Conferência da Associação Internacional para Pesquisas de Uso do Tempo (IATUR), Rio de Janeiro, Brasil.

Moraes, M. L. Q. (1985). Mulheres em movimento. São Paulo: Conselho da Condição Feminina.

Moraes, M. L. Q. (1990). A experiência feminista dos anos setenta. São Paulo: UNESP.

Napolitano, M. (2014). 1964: história do regime militar brasileiro. São Paulo: Contexto.

Nós Mulheres [São Paulo] (1976), № 1.

Pedro, J. M. (2006). Narrativas fundadoras do feminismo: poderes e conflitos (1970-1978). Revista Brasileira de História, 26(52), 249-272. 
Pedro, J. M. (2011). Relações de gênero como categoria transversal na historiografia contemporânea. Topoi, 12(22), 271-283.

Pollak, M. (1989). Memória, esquecimento, silêncio. Estudos históricos, 2(3), 3-15.

Prado, D. (1979). Ser esposa - a mais antiga profissão. São Paulo: Brasiliense.

Revista gênero (2006) 6(2) y 7(1). Recuperada de http://www.revistagenero.uff.br/index.php/revistagenero/issue/vi ew/27/showToc

Sardenberg, C. M. B. (2002). Da crítica feminista à ciência. Uma ciência feminista? (pp. 89-120). En A. A. A. Costa e C. M. B. Sardenberg, Cecília Maria Bacellar (Orgs.), Feminismo, ciência e tecnologia. Salvador de Bahía: REDOR/ NEIM-FFCH/UFBA.

Sarti, C. A. (2004). O feminismo brasileiro desde os anos 1970: revisitando uma trajetória. Estudos feministas, 12(2), $35-50$.

Scott, J. W. (2002). A cidadã paradoxal: as feministas francesas e os direitos do homem. Florianópolis: Ed. Mulheres.

Scott, J. W. (2005). O enigma da igualdade. Estudos feministas, 13(1), 11-30.

Silva, C. (1979). Na luta para se afirmar a mulher tem que ser... Super mulher! Claudia, 210, 168-169.

Silveira, D. (7 de diciembre de 2017). Mulheres passam o dobro do tempo dos homens com tarefas domésticas, aponta IBGE. O Globoonline. Recuperado de https://g1.globo.com/economia/noticia/mulheres-passam-o-dobro-do-t empo-dos-homens-com-tarefas-domesticas-aponta-ibge.ghtml

Tiburi, M. (21 de setembro de 2016). PartidA, um movimento feminista feito de alegria política. Cult. Recuperado de https://revistacult.uol.com.br/home/partida-um-movimento-feminista-feito-de-alegria-politica

Vasconcellos, B. M. y Lima, M. M. T. (2016). Fanny Tabak e os primeiros passos dos estudos sobre Ciência, Tecnologia e Gênero no Brasil. Redes, 22(43),13-32.

Wolff, C. S. (2015).Mulheres de luta:feminismo e esquerdas no Brasil (1964-1985). Projeto de pesquisa. CAPES: Edital 12/2015, Memórias Brasileiras: Conflitos Sociais.

Wolff, C. S., Crescêncio, C. L. y Pedro, J. M. (2016). Ondas, mitos e contradições: feminismos em tempos de ditaduras no Cone Sul (pp. 53-70). En M. A. Monte Rocha (Org.), Feminismos plurais. Fortaleza, Brasil: Expressão Gráfia e Editora.

\section{FUENTES}

Entrevista a Fani Miranda Tabak, en Florianópolis, de fecha 01/08/2017, realizada por Soraia Carolina de Mello, filmada por Elaine Schmitt y transcripta por Luana Balieiro Cosme.

Entrevista a Hildete Pereira de Melo Hermes de Araujo, en Florianópolis, de fecha 04/08/2017, realizada por Soraia Carolina de Mello, filmada por Elaine Schmitt y transcripta por Alina Nunes y Valéria Machado.

Entrevista a Luci Teresinha Choinacki, en Florianópolis, de fecha 25/09/2017, realizada por Soraia Carolina de Mello, filmada por Elaine Schmitt y transcripta por Jeferson Ramos.

\section{Notas}

1 "O SOS CORPO - Instituto Feminista para a Democracia es una organización no gubernamental, fundada en la década del ' 80 en la ciudad de Recife, Pernambuco, Brasil.” Recuperado de: http://soscorpo.org/quem-somos/

2 Breve reportaje que aborda los femicidios en el estado de Minas Gerais y sus repercusiones durante el inicio de los años 1980,se puede acceder al mismo en Guimarães, Katia. (12 de dezembro de 2017). Mais de 40 anos após Ângela Diniz, Minas continua campeã em feminicídios. Socialista Morena. Recuperado de http://www.socialistamorena.com.br/mai s-de-40-anos-apos-morte-de-angela-diniz-minas-continua-campea-em-feminicidios

3 Traducción propia. 